\title{
Mechanistic Significance of Intermittent Pulmonary Vein Tachycardia in Patients with Atrial Fibrillation
}

\author{
HAKAN ORAL, M.D., MEHMET ÖZAYDIN, M.D., HIROSHI TADA, M.D., \\ AMAN CHUGH, M.D., CHRISTOPH SCHARF, M.D., SOHAIL HASSAN, M.D., \\ STEVE LAI, M.D., RADMIRA GREENSTEIN, M.D., FRANK PELOSI, JR., M.D., \\ BRADLEY P. KNIGHT, M.D., S. ADAM STRICKBERGER, M.D., \\ and FRED MORADY, M.D.
}

From the Division of Cardiology, Department of Internal Medicine, University of Michigan, Ann Arbor, Michigan

\begin{abstract}
Pulmonary Vein Tachycardia. Introduction: The significance of intermittent tachycardia within a pulmonary vein $(\mathrm{PV})$ during an episode of atrial fibrillation $(\mathrm{AF})$ is unclear. The aim of this study was to determine the role that intermittent $\mathrm{PV}$ tachycardias play in AF.

Methods and Results: In 56 patients with AF, segmental ostial ablation guided by PV potentials was performed to isolate the PVs. The characteristics of intermittent PV tachycardias and the inducibility of AF before and after PV isolation were analyzed prospectively. During AF, a PV tachycardia (mean cycle length $130 \pm 30 \mathrm{msec}$ ) with exit block to the left atrium was present in $93 \%$ of left superior, $80 \%$ of left inferior, 73\% of right superior, and $7 \%$ of right inferior PVs. The site of shortest cycle length during AF alternated between the PVs and left atrium 1 to 13 times per minute. Complete isolation was achieved in 168 (94\%) of 178 targeted PVs. In 99\% of PVs, tachycardia resolved upon isolation. AF was persistent before and after $\mathrm{PV}$ isolation in $100 \%$ and $27 \%$ of patients, respectively $(P<0.001)$.

Conclusion: Intermittent bursts of tachycardia are observed within multiple PVs during persistent AF in a majority of patients. After PV isolation, $\mathrm{PV}$ tachycardias almost always resolve, and $\mathrm{AF}$ is less likely to be inducible or persistent. These observations suggest a dynamic interplay between the atria and PVs, with intermittent bursts of PV tachycardia being dependent on left atrial input and with the probability of persistent AF diminishing when PV tachycardias are eliminated by $\mathbf{P V}$ isolation. ( $\mathrm{J}$ Cardiovasc Electrophysiol, Vol. 13, pp. 645-650, July 2002)
\end{abstract}

atrial fibrillation, radiofrequency catheter ablation, pulmonary vein

\section{Introduction}

Premature depolarizations arising in the pulmonary veins (PVs) may trigger atrial fibrillation (AF). ${ }^{1}$ In addition, intermittent tachycardias may be observed within the PVs during AF, but their significance is unclear. ${ }^{2}$ The aim of this study was to determine the prevalence of intermittent tachycardia in the PVs during AF, and the effect of PV isolation on PV tachycardia and on the inducibility of persistent AF.

\section{Methods}

\section{Patient Characteristics}

The subjects of this prospective study were 56 consecutive patients with symptomatic AF who underwent PV isolation. Their characteristics are listed in Table 1. A mean of $2.4 \pm 1.3$ antiarrhythmic drugs had failed to suppress AF. The patients were asked to maintain a log of the number and duration of episodes of symptomatic AF during a 2-month period before the ablation procedure.

Supported in part by the Ellen and Robert Thompson Atrial Fibrillation Research Fund.

Address for correspondence: Hakan Oral, M.D., Department of Cardiology, University of Michigan, 1500 E. Medical Center Drive, B1F245, Ann Arbor, MI 48109-0022.Fax: 734-936-7026; E-mail: oralh@umich.edu

Manuscript received 11 February 2002; Accepted for publication 1 May 2002.

\section{Electrophysiologic Study}

All patients provided written, informed consent. A femoral vein approach was used. A quadripolar electrode catheter (EP Technologies, Inc., San Jose, CA, USA) was positioned in the distal coronary sinus and used for atrial pacing. Transseptal catheterization was performed, and a deflectable, decapolar catheter with a distal ring configuration (Lasso catheter, Biosense Webster, Diamond Bar, CA, USA) and a deflectable, quadripolar 7-French catheter with 2-5-2 $\mathrm{mm}$ interelectrode spacing and a 4-mm distal electrode with an embedded thermistor (EP Technologies, Inc.) were inserted into the left atrium. Heparin was administered intravenously to maintain an activated clotting time of 250 to 350 seconds. Angiograms of the left superior (LS), right superior (RS), and left inferior (LI) PVs were performed. Bipolar electrograms were filtered at bandpass settings of 30 to $500 \mathrm{~Hz}$ and recorded digitally (EPMedSystems, Inc., Mount Arlington, NJ, USA).

\section{Study Protocol}

$\mathrm{AF}$ was present at the onset of the procedure in 25 patients $(45 \%)$ and was induced by atrial pacing at a cycle length of $200 \mathrm{msec}$ in 31 patients (55\%). The Lasso catheter was positioned sequentially in the LS, LI, and RS PVs, within $5 \mathrm{~mm}$ of the ostium. The Lasso catheter often could not be positioned within the right inferior (RI) PV, in which case this vein was mapped with the ablation catheter.

The ablation catheter was positioned against the left 
TABLE 1

Characteristics of the Subjects

\begin{tabular}{lc}
\hline Age (years) & $53 \pm 12(27-73)$ \\
Males/females & $45 / 11$ \\
Paroxysmal AF/persistent AF & $49 / 7$ \\
Duration of AF (years) & $7.1 \pm 6.7$ \\
Frequency of episodes of AF (per month) & $15 \pm 12$ \\
Left ventricular ejection fraction & $0.54 \pm 0.05(0.35-0.60)$ \\
Left atrial size (mm) & $39.6 \pm 4.2$ \\
Structural heart disease & 1 \\
$\quad$ Ischemic heart disease & 3 \\
Nonischemic cardiomyopathy & 1 \\
Mitral valve prolapse & 51 \\
None &
\end{tabular}

Data are shown as mean \pm SD. Range is shown in parenthesis.

$\mathrm{AF}=$ atrial fibrillation.

atrial wall, in proximity to the PV in which the Lasso catheter was positioned. Electrograms were recorded simultaneously from the PV, left atrium, and coronary sinus for at least 2 minutes. During AF, the initial rapid depolarization indicated local activation. To account for far-field and fragmented electrograms, only electrograms that were $>10 \%$ of the amplitude of the largest electrogram and at least $50 \mathrm{msec}$ apart were analyzed. When the cycle length inside the PV was shorter than in the left atrium near that vein, this was considered evidence of a PV tachycardia (Fig. 1). In contrast, when the left atrial cycle length just outside the PV was shorter than within the PV, this was considered to be indicative of passive activation of the PV from the left atrium (Fig. 1).

After sinus rhythm was restored by transthoracic cardioversion, $\mathrm{PV}$ isolation was performed during sinus rhythm or coronary sinus pacing by delivery of radiofrequency energy at ostial sites. At a minimum, the PVs that displayed tachycardias that had a cycle length shorter than in the adjacent left atrium were targeted for isolation. To minimize the probability of recurrent AF, whenever feasible, the LS, LI, and RS PVs were isolated. The RI PV was targeted for isolation if PV potentials were identified within this vein.

In $20(35 \%)$ of 56 patients, ibutilide $1 \mathrm{mg}$ or amiodarone $300 \mathrm{mg}$ was administered intravenously to prevent immediate recurrence of AF (IRAF) after cardioversion. However, in 6 patients $(11 \%)$, IRAF was not prevented by these drugs. In these patients, ablation was performed during AF.

Radiofrequency energy was delivered at the PV ostium as individual 30- to 60-second applications, with a target temperature of $52^{\circ} \mathrm{C}$ and a maximum power output of 30 to 35 W (model EPT-1000-TC, EP Technologies, Inc.). Elimination of all ostial PV potentials and complete entrance block into the PV were considered indicative of complete electrical isolation of the PV (Fig. 2).

After complete isolation of all targeted PVs, AF induction was attempted by 5 -second bursts of atrial pacing at cycle lengths of 220 to $180 \mathrm{msec}$ in 10 -msec decrements. AF that terminated spontaneously within 5 minutes was considered nonsustained. If induced AF was nonsustained, it was reinduced at least twice to confirm spontaneous termination within 5 minutes. To determine whether AF was inducible, rapid atrial pacing was performed after infusion of dobutamine 10 to $20 \mu \mathrm{g} / \mathrm{min} / \mathrm{kg}$ or isoproterenol 2 to $4 \mu \mathrm{g} / \mathrm{min}$.

\section{Follow-Up}

The patients were seen in an outpatient clinic 4 to 6 weeks and 3 to 4 months after the ablation procedure. All patients were asked to keep a log of the duration and frequency of their symptoms. All patients who reported symptoms were given an event monitor to document the cause of symptoms.

\section{Statistical Analysis}

Continuous variables are expressed as mean \pm 1 SD. Comparisons of tachycardia cycle lengths within the PVs and left atrium were performed using a paired $t$-test. Tachy-

\section{A PV tachycardia with exit block}

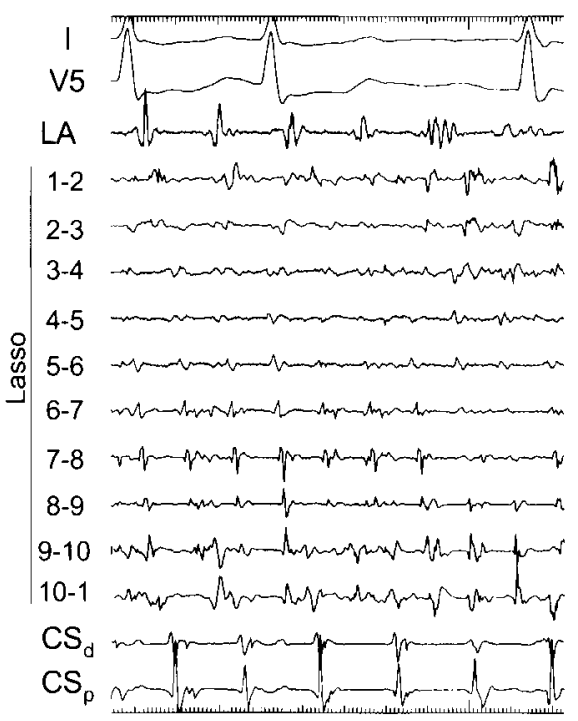

B
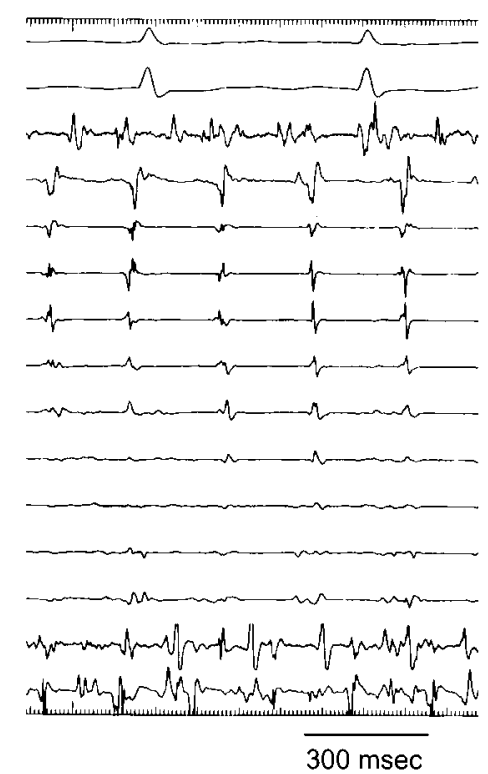

Figure 1. Alternation in the site of shortest cycle length between a pulmonary vein $(P V)$ and the adjacent left atrium. Shown are leads $I$ and $V_{5}$ and intracardiac electrograms recorded from the left atrial (LA), Lasso, and coronary sinus (CS) catheters. The Lasso catheter was positioned within $5 \mathrm{~mm}$ of the ostium of the left superior PV, and the LA catheter was positioned against the $L A$ wall adjacent to the left superior PV ostium. (A) PV tachycardia with a mean cycle length of $113 \mathrm{msec}$, which is shorter than the mean cycle length of $180 \mathrm{msec}$ in the left atrium. (B) Recordings obtained a few seconds later, during the same episode of $A F$. Now the cycle length within the left atrium, $112 \mathrm{msec}$, is shorter than in the PV, 180 msec, consistent with entrance block into the PV. $C S_{d}=$ distal electrode pair of the coronary sinus catheter; $C S_{P}=$ proximal electrode pair of the coronary sinus catheter. 
Figure 2. Example of complete isolation of a pulmonary vein $(P V)$. Shown are leads $I$ and $V_{5}$ and intracardiac electrograms recorded from the left atrial (LA), Lasso, and coronary sinus (CS) catheters. The Lasso catheter was positioned within $5 \mathrm{~mm}$ of the ostium of the left superior PV (LSPV) and the LA catheter was positioned against the LA wall adjacent to the LS PV ostium. After PV isolation (B), the ablation catheter was moved to the right superior $P V$ $(R S P V)$, but the Lasso catheter was not moved from the LS PV. Before isolation there was a PV tachycardia with exit block to the left atrium (A). After isolation of the $P V$, no electrograms are recorded within the LS PV during AF (B). Abbreviations as in Figure 1.

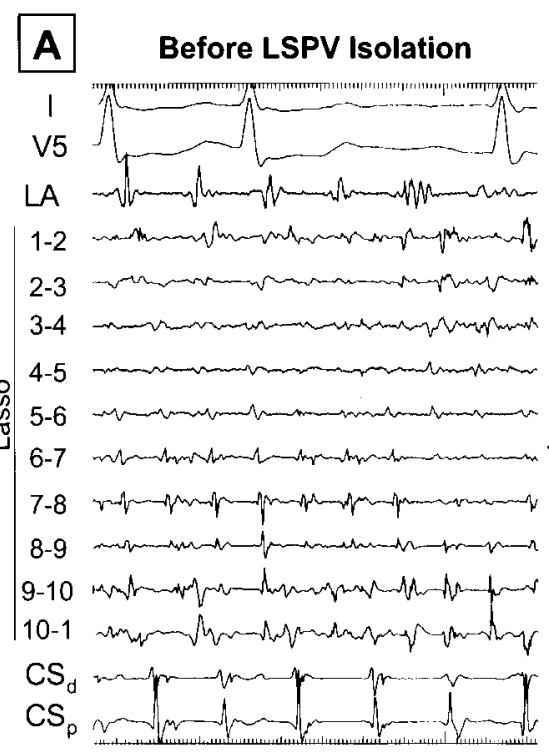

cardia cycle lengths among the PVs were compared by analysis of variance. Categorical variables were compared with the Chi-square test. Kaplan-Meier analysis with log rank test was used to compare the probability of freedom from symptomatic AF in the patients who did and those who did not have inducible persistent $\mathrm{AF}$ after $\mathrm{PV}$ isolation. $\mathrm{P}<$ 0.05 were considered statistically significant.

\section{Results}

\section{Prevalence of PV Tachycardias Before PV Isolation}

A PV tachycardia was present during AF in $52(93 \%)$ of 56 LS PVs, 41 (73\%) of 56 RS PVs, 45 (80\%) of 54 LI PVs, and 4 (7\%) of 56 RI PVs. The prevalence of PV tachycardia was similar during spontaneous and induced AF (Fig. 3). PV tachycardia was observed less frequently in the RI PV than in the other three PVs ( $<<0.001$; Fig. 3). PV tachycardia was present in all four PVs during the same episode

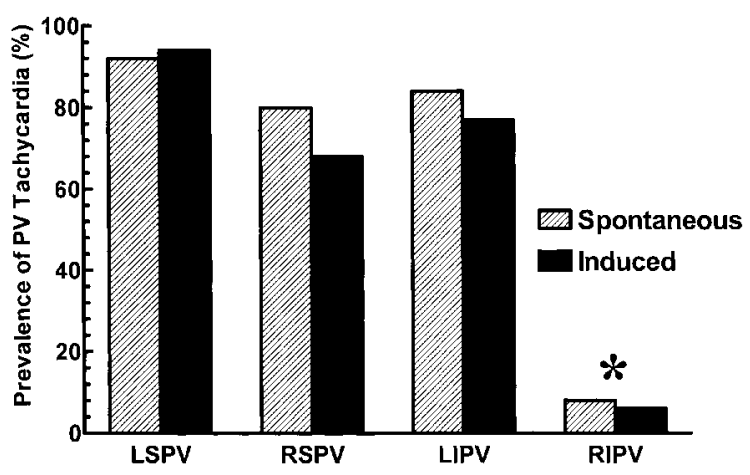

Figure 3. Prevalence of pulmonary vein $(P V)$ tachycardia with exit block to the left atrium during spontaneous and induced episodes of atrial fibrillation (AF). There were no significant differences in the prevalence of exit block between spontaneous and induced episodes of AF. The prevalence of $P V$ tachycardia was lower in the right inferior pulmonary vein $(R I P V)$ than in the other PVs. LIPV = left inferior pulmonary vein; $L S P V=$ left superior pulmonary vein; $R S P V=$ right superior pulmonary vein. $* P<0.001$. of $\mathrm{AF}$ in 1 patient (2\%), in three PVs in 38 patients (68\%), in two PVs in 10 patients (18\%), and in one PV in 4 patients (7\%).

\section{Cycle Length Within the PV and Left Atrium During $\boldsymbol{A F}$}

During AF, PV tachycardias occurred as intermittent bursts with a mean duration of $1.2 \pm 0.6$ seconds (Fig. 1). During PV tachycardia, the mean cycle length within the PV $(130 \pm 30 \mathrm{msec})$ was significantly shorter than the mean cycle length within the adjacent left atrium $(182 \pm 29 \mathrm{msec}$, $\mathrm{P}<0.001)$. There was no significant difference in tachycardia cycle length among the PVs (Table 2).

Between the bursts of PV tachycardia, the cycle length measured at the adjacent left atrium (134 $\pm 34 \mathrm{msec})$ sometimes was shorter than within the PV $(190 \pm 45 \mathrm{msec}$, $\mathrm{P}<0.001$, Table 3 ). The site of shortest cycle length alternated between the PV and adjacent left atrium at a median of 3 times per minute (range 1 to 13 ).

\section{PV Isolation}

All four PVs were targeted for isolation in 12 patients, three PVs were targeted in 43 patients, and two PVs were targeted in 1 patient. Electrical isolation was complete in 51 (91\%) of 56 LS PVs, 54 (96\%) of the 56 RS PVs, 51 (94\%) of $54 \mathrm{LI}$ PVs, and $12(100 \%)$ of 12 RI PVs in which

\section{TABLE 2}

Pulmonary Vein Tachycardia Cycle Lengths and Simultaneous Cycle Lengths in the Adjacent Left Atrium When the Shortest Cycle Length Occurred in the Pulmonary Vein

\begin{tabular}{lcccc}
\hline & Left Superior & Right Superior & Left Inferior & Right Inferior \\
\hline PV & $127 \pm 29$ & $135 \pm 31$ & $129 \pm 30$ & $123 \pm 26$ \\
LA & $186 \pm 30$ & $180 \pm 25$ & $180 \pm 31$ & $178 \pm 25$ \\
P value & $<0.001$ & $<0.001$ & $<0.001$ & $<0.001$ \\
\hline
\end{tabular}

Cycle lengths are expressed as mean $\pm \mathrm{SD}$, in milliseconds.

$\mathrm{LA}=$ left atrium, adjacent to corresponding $\mathrm{PV} ; \mathrm{PV}=$ pulmonary vein. 


\section{TABLE 3}

Pulmonary Vein Tachycardia Cycle Lengths and Simultaneous Cycle Lengths in the Adjacent Left Atrium When the Shortest Cycle Length Occurred in the Left Atrium

\begin{tabular}{lcccc}
\hline & Left Superior & Right Superior & Left Inferior & Right Inferior \\
\hline PV & $198 \pm 44$ & $189 \pm 49$ & $181 \pm 42$ & $179 \pm 19$ \\
LA & $137 \pm 33$ & $138 \pm 39$ & $127 \pm 32$ & $125 \pm 20$ \\
P value & $<0.001$ & $<0.001$ & $<0.001$ & $<0.01$ \\
\hline
\end{tabular}

Cycle lengths are expressed as mean $\pm \mathrm{SD}$, in milliseconds.

$\mathrm{LA}=$ left atrium, adjacent to corresponding PV; PV = pulmonary vein.

isolation was attempted. All targeted PVs were completely isolated in $46(82 \%)$ of the 56 patients.

\section{Inducibility of AF Before and After Complete PV Isolation}

All 56 patients had either spontaneous or inducible persistent $\mathrm{AF}$ before $\mathrm{PV}$ isolation. After PV isolation, AF was inducible but nonsustained in 41 patients (73\%), and inducible and persistent for $\geq 5$ minutes in 15 patients (27\%; Fig. 4). The prevalence of persistent AF was significantly lower after than before PV isolation $(27 \%$ vs $100 \%$; $<<0.001)$. The mean duration of the nonsustained episodes of AF after ablation was $0.73 \pm 1.49$ minutes, and the mean duration of the persistent episodes of induced AF was $16.3 \pm 9.6$ minutes, up to the point of cardioversion. There was no significant difference in the prevalence of inducible AF after PV isolation between patients who received ibutilide or amiodarone (43\%) and those who did not $(29 \% ; \mathrm{P}=0.5)$.

$\mathrm{AF}$ was inducible and persistent in the 1 patient who did not have complete isolation of any PV, in $2(40 \%)$ of 5 patients with two PVs isolated, in $8(21 \%)$ of 38 patients with three PVs isolated, and in $4(36 \%)$ of 11 patients with all four $\mathrm{PVs}$ isolated $(\mathrm{P}=0.3)$.

\section{PV Rhythms After PV Isolation}

Among the 168 isolated PVs, 11 (7\%) displayed a PV bradycardia that had a mean cycle length of $3.2 \pm 1.3$ seconds and $2(1 \%)$ displayed a PV tachycardia that had at a mean cycle length of $129 \mathrm{msec}$ (Fig. 5). These rhythms were dissociated from the left atrium. Twelve of the 13 spontaneous PV rhythms disappeared within 10 minutes after PV isolation. No spontaneous rhythms were recorded in the remaining PVs after complete isolation (Fig. 2).

After complete PV isolation and resolution of the spontaneous PV rhythms, a PV tachycardia was never observed during induced $\mathrm{AF}$ and was never inducible by rapid atrial pacing or dobutamine infusion.

\section{Inducibility of AF and Recurrence of Symptomatic AF}

At a mean of $122 \pm 77$ days of follow-up, 34 (61\%) of the 56 patients had no recurrences of symptomatic AF, and 22 patients (39\%) had a recurrence of $\geq 1$ episode of AF. Among these 22 patients, 4 had no further episodes of AF with an antiarrhythmic agent that was previously ineffective, and 4 had $>90 \%$ improvement in the frequency of AF episodes in the absence of antiarrhythmic drug therapy.

Patients in whom persistent AF was not inducible after $\mathrm{PV}$ isolation remained free of symptomatic AF or had $>90 \%$ reduction in the incidence of symptomatic AF significantly more often than patients in whom persistent $\mathrm{AF}$ was inducible ( $\mathrm{P}=0.04$; Fig. 6 ).

\section{Discussion}

\section{Main Findings}

The main findings of this study are as follows. (1) During $\mathrm{AF}$, whether spontaneous or induced, tachycardia commonly is observed in multiple PVs. (2) PV tachycardia with a cycle length shorter than in the adjacent atrium usually occurs as intermittent bursts that are separated by periods of passive activation of the PVs. (3) PV tachycardias depend on input to the PVs from the left atrium and almost always are no longer present after PV isolation. (4) After PV isolation, when PV tachycardias are no longer present, inducible AF is less likely to be sustained. (5) PV bradycardias and tachycardias that are dissociated from sinus

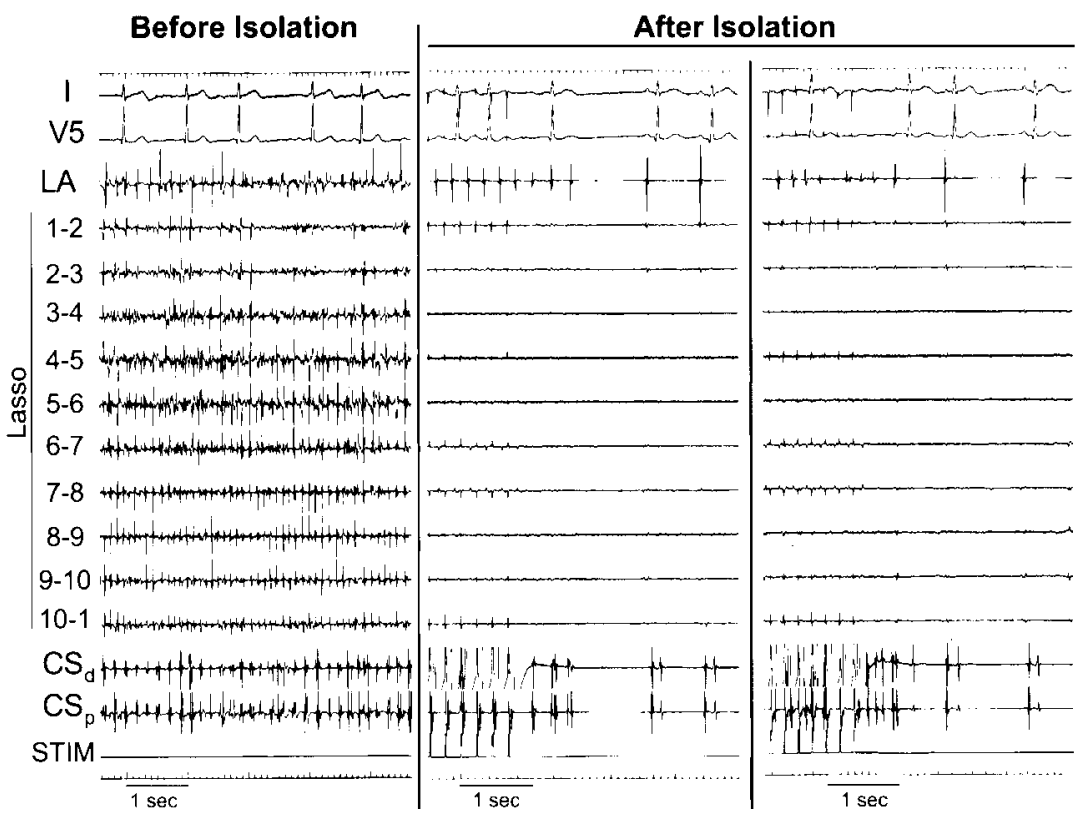

Figure 4. Inducibility of atrial fibrillation $(A F)$ before and after pulmonary vein $(P V)$ isolation. Format and catheter positions as in Figure 1. Before PV isolation, persistent $A F$ was inducible by atrial pacing at a cycle length of $200 \mathrm{msec}$. After isolation of the left superior, left inferior, and right superior PVs, persistent AF was no longer inducible. Two of several induction attempts are shown. STIM = stimulation marker. Other abbreviations as in Figure 1. 
Figure 5. Pulmonary vein (PV) tachycardia that persisted after isolation of the left superior PV. Format and catheter positions as in Figure 1. Before isolation, there was a PV tachycardia with a shorter cycle length than in the adjacent left atrium. Radiofrequency energy was delivered at the ostial sites at which PV tachycardia was recorded. After isolation, short bursts of PV tachycardia with complete exit block (arrows) were still recorded within the vein, but they disappeared several minutes later. The potentials that are simultaneous with the $P$ waves are atrial potentials. STIM = stimulation marker. Other abbreviations as in Figure 1.

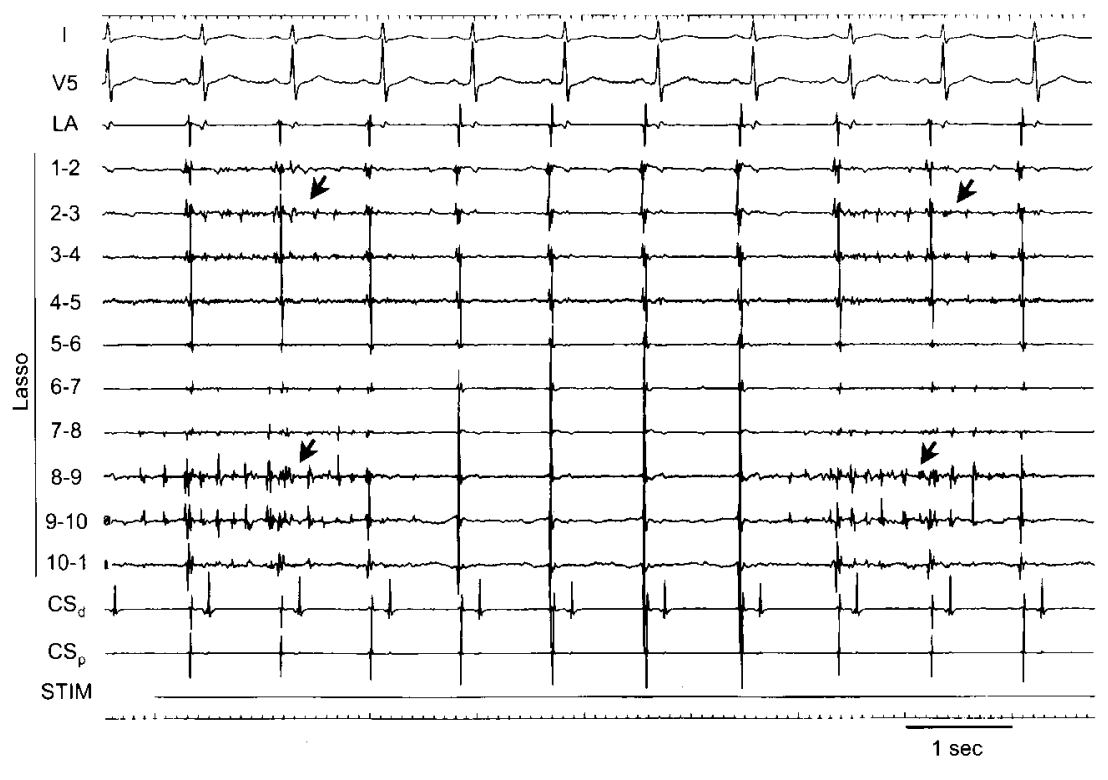

rhythm occur in $7 \%$ and $1 \%$ of isolated PVs, respectively, and usually disappear within 10 minutes after PV isolation, resulting in a quiescent PV. (7) Recurrences of symptomatic $\mathrm{AF}$ after $\mathrm{PV}$ isolation are less likely when persistent $\mathrm{AF}$ is not inducible by rapid pacing.

These findings suggest that there may be a dynamic interplay between the left atrium and PVs during $\mathrm{AF}$, with PV tachycardia depending on input from the atrium, and with the likelihood of inducible persistent AF being markedly diminished by PV isolation and elimination of the intermittent bursts of PV tachycardia. Therefore, the PVs may play an important role in the maintenance of $\mathrm{AF}$, above and beyond serving as a possible source of arrhythmias that initiate $\mathrm{AF}$.

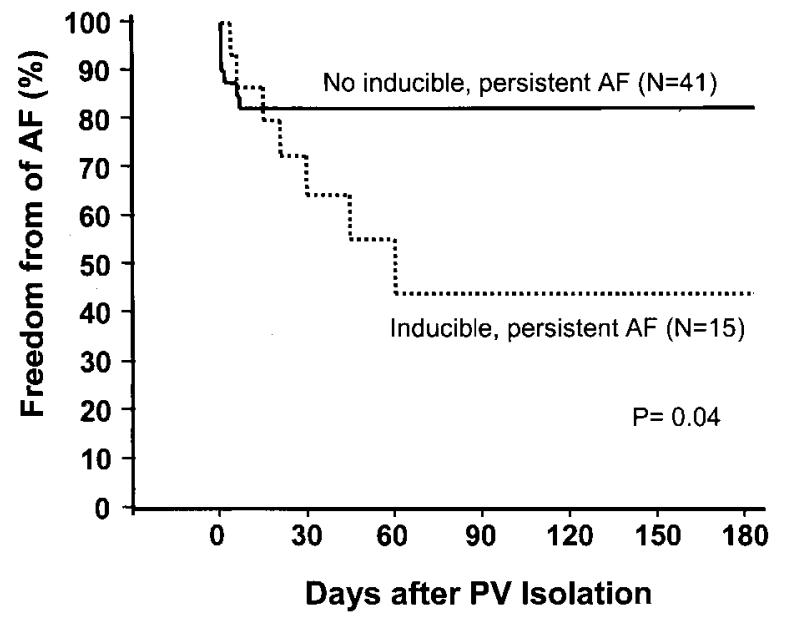

Figure 6. Freedom from recurrence of atrial fibrillation $(A F)$ in patients who did and those who did not have inducible persistent AF after pulmonary vein $(P V)$ isolation. Patients who did not have inducible persistent $A F$ after PV isolation (solid line) were more likely to have a successful outcome (either no AF recurrence or $>90 \%$ improvement in the frequency of symptoms) than patients who had inducible persistent $A F$ (dashed line; $P=0.04)$. Number of patients in each group is shown in parentheses.

\section{Role of PVs in AF}

Prior studies demonstrated that premature depolarizations that initiate AF may arise in a PV. ${ }^{1,3}$ Therefore, it could be argued that the clinical benefit of PV isolation in the present study was attributable simply to elimination of the initiating triggers that arose in the PVs. However, elimination of the initiating triggers should not have affected the inducibility of AF by rapid pacing and cannot account for the association found in this study between the inducibility of AF and clinical outcome.

The present study suggests an additional role that the PVs may play in AF, namely maintenance of AF by intermittent bursts of tachycardia. AF can reliably be induced by rapid atrial pacing, and the tendency toward spontaneous conversion to sinus rhythm can be prevented by intermittent bursts of rapid pacing. ${ }^{4}$ The observations in this study suggest that the intermittent bursts of PV tachycardia that occur during AF may play the same role in sustaining AF as do intermittent bursts of rapid atrial pacing. This would explain why persistent AF was inducible much less often after than before PV isolation.

Sustained AF was inducible by rapid atrial pacing despite PV isolation in $27 \%$ of patients in this study. Therefore, it is clear that intermittent bursts of PV tachycardia are not a requirement for sustained AF. It is likely that there are several factors that determine the duration of an episode of $\mathrm{AF},{ }^{5}$ and it is possible that intermittent $\mathrm{PV}$ tachycardia is one of these factors.

Almost all of the patients in this study had idiopathic, paroxysmal AF. However, the results of surgical studies suggest that the PVs may play a role in sustaining AF even in patients with mitral valve disease and dilated atria. Melo et al. ${ }^{6}$ reported that AF was no longer present in a majority of patients when PV isolation was performed at the time of mitral valve surgery. This surgical experience is consistent with a role of PV tachycardia in maintaining AF even when the atria are dilated and have undergone structural remodeling. 


\section{Mechanisms of PV Tachycardia}

In this study, PV tachycardias were almost always immediately abolished by PV isolation. Because prior studies demonstrated that arrhythmogenic PV foci often are located $>1 \mathrm{~cm}$ in from the ostium, it is unlikely that the ostial applications of RF energy in the present study directly ablated the foci that were generating the PV tachycardias. ${ }^{1,2}$ Moreover, a PV tachycardia that was still present after isolation became progressively shorter in duration and resolved within several minutes.

These observations suggest that PV tachycardias may depend on input from the left atrium. If so, this would suggest that PV tachycardias during AF are caused by either triggered activity or reentry and not by abnormal automaticity, because the latter would not be expected to depend on passive activation of the PV from the left atrium. Consistent with the mechanism of triggered activity is the observation in guinea pigs that disconnection of the PVs from the left atrium eliminates ouabain-induced triggered activity within the PVs. ${ }^{7}$

\section{Study Limitations}

A limitation of this study is that $35 \%$ of patients received an antiarrhythmic drug to prevent IRAF after cardioversion. It is possible that a persistent effect of these drugs influenced the inducibility of sustained AF after PV isolation. However, because the findings after PV isolation were similar in the patients who did and those who did not receive an antiarrhythmic drug, it seems unlikely that the drugs were a significant confounding variable.

A second limitation of this study is that the inducibility of AF was assessed only after isolation of all targeted PVs, not after isolation of individual PVs. Therefore, the possibility that the decreased susceptibility to induction of sus- tained AF was attributable to a single dominant PV instead of multiple PVs cannot be ruled out.

\section{Conclusion}

Intermittent bursts of PV tachycardia may be one of the factors that sustains an episode of AF. This suggests a role for $\mathrm{PV}$ isolation even when the initial trigger for $\mathrm{AF}$ does not originate within a PV. In addition, by eliminating one of the factors that may perpetuate an episode of $\mathrm{AF}$, even when $\mathrm{PV}$ isolation does not abolish all episodes of AF, it may be helpful by increasing the probability of spontaneous conversion to sinus rhythm or by increasing the responsiveness to previously ineffective antiarrhythmic drugs.

\section{References}

1. Haissaguerre M, Jais P, Shah DC, Takahashi A, Hocini M, Quiniou G, Garrigue S, Le Mouroux A, Le Metayer P, Clementy J: Spontaneous initiation of atrial fibrillation by ectopic beats originating in the pulmonary veins. N Engl J Med 1998;339:659-666.

2. Tse HF, Lau CP, Kou W, Pelosi F, Oral H, Kim M, Michaud GF, Knight BP, Moscucci M, Strickberger SA, Morady F: Prevalence and significance of exit block during arrhythmias arising in pulmonary veins. J Cardiovasc Electrophysiol 2000;11:379-386.

3. Chen SA, Hsieh MH, Tai CT, Tsai CF, Prakash VS, Yu WC, Hsu TL, Ding YA, Chang MS: Initiation of atrial fibrillation by ectopic beats originating from the pulmonary veins: Electrophysiological characteristics, pharmacological responses, and effects of radiofrequency ablation. Circulation 1999;100:1879-1886

4. Wijffels MC, Kirchhof CJ, Dorland R, Allessie MA: Atrial fibrillation begets atrial fibrillation. A study in awake chronically instrumented goats. Circulation 1995;92:1954-1968.

5. Allessie MA, Boyden PA, Camm AJ, Kleber AG, Lab MJ, Legato MJ, Rosen MR, Schwartz PJ, Spooner PM, Van Wagoner DR, Waldo AL: Pathophysiology and prevention of atrial fibrillation. Circulation 2001; 103:769-777.

6. Melo J, Adragao P, Neves J, Ferreira MM, Pinto MM, Rebocho MJ, Parreira L, Ramos T: Surgery for atrial fibrillation using radiofrequency catheter ablation: Assessment of results at one year. Eur J Cardiothorac Surg 1999;15:851-854.

7. Cheung DW: Pulmonary vein as an ectopic focus in digitalis-induced arrhythmia. Nature 1981;294:582-584. 